\section{Drs. Kang and Ju reply}

\section{To the Editor:}

Dr. Dogan and Dr. Agilli raised several issues ${ }^{1}$ about our article ${ }^{2}$. The first issue was that the study population needed to be clearly defined, because comorbid diseases or medications might affect serum uric acid (SUA) levels. To address this issue, we performed medical chart review, which showed that the number of patients with hypertension was fewer than $5(3.3 \%)$. The same number of patients had dyslipidemia. And there was only 1 patient with diabetes who was taking metformin. None of the patients had currently or a history of cardiovascular disease, nonalcoholic fatty liver, peripheral arterial disease, multiple sclerosis, or Parkinson's disease (Table 1). Our study subjects were relatively young and lean patients with a mean age of 32 years and mean BMI of $23.4 \mathrm{~kg} / \mathrm{m}^{2}$. This may explain why our subjects had few comorbid conditions or medications. The second issue was about blood sampling time. It was reported that SUA level was affected by diurnal variation. In our study, morning blood samples were obtained after $12 \mathrm{~h}$ of fasting and were subsequently analyzed at a certified laboratory at the Seoul St. Mary's hospital.

Table 1. The comorbidity and medications in patients with ankylosing spondylititis $(\mathrm{n}=150)$.

\begin{tabular}{lcc}
\hline Comorbid Disease & Medication & No. $(\%)$ \\
\hline Diabetes melitus & & $1(0.7)$ \\
Hypertension & Metformin & $1(0.7)$ \\
& & $5(3.3)$ \\
& CCB & $4(2.7)$ \\
ACEi & $0(0)$ \\
& ARB & $2(1.3)$ \\
& & $5(3.3)$ \\
CVDslipidemia & Fenofibrate & $0(0)$ \\
Nonalcoholic fatty liver & Simvastatin & $2(1.3)$ \\
Peripheral arterial disease & Atorvastatin & $3(2.0)$ \\
Multiple sclerosis & & $0(0)$ \\
Parkinson's disease & & $0(0)$ \\
& & $0(0)$ \\
\hline
\end{tabular}

CVD: cardiovascular disease; CCB: calcium channel blocker; ACEi: angiotensin-converting enzyme inhibitor; ARB: angiotensin receptor blocker.
We appreciate Dr. Dogan's constructive comments, which provided us with the opportunity to thoroughly review the medical history of our subjects. This review revealed that only a small proportion of our subjects had comorbid conditions or medications that could affect SUA. Therefore, there is little chance that comorbidities or medications significantly affected the SUA of our study population. As Dr. Dogan mentioned, this clarification made our article clearer and more informative.

KWI YOUNG KANG, MD, PhD, Division of Rheumatology, Department of Internal Medicine, Incheon Saint Mary's Hospital, College of Medicine, The Catholic University of Korea, Incheon; JI HYEON JU, MD, PhD, Division of Rheumatology, Department of Internal Medicine, Seoul St. Mary's Hospital, College of Medicine, The Catholic University of Korea, Seoul, South Korea. Address correspondence to Dr. J.H. Ju, Division of Rheumatology, Department of Internal Medicine, Seoul St. Mary's Hospital, College of Medicine, The Catholic University of Korea, \#505, Banpo-Dong, Seocho-Gu, Seoul, South Korea.

E-mail address: juji@catholic.ac.kr

\section{REFERENCES}

1. Dogan T, Agilli M. Assessment of serum uric acid in young male patients with ankylosing spondylitis. J Rheumatol 2016;43:678.

2. Kang KY, Hong YS, Park SH, Ju JH. Low levels of serum uric acid increase the risk of low bone mineral density in young male patients with ankylosing spondylitis. J Rheumatol 2015;42:968-74.

J Rheumatol 2016;43:3; doi:10.3899/jrheum.151268 